\title{
Phase Synchronization Underlying the Mutual Inductance Effect on Chua's Circuits
}

\author{
Feng-Wei Tsai, ${ }^{1}$ Tzu-Pin Yu, ${ }^{1}$ Ming-Chi Lu, ${ }^{1}$ Chen-An Chan, ${ }^{1}$ Yan-Lin Zhong, \\ Tang-Yi Tsai, ${ }^{2}$ Chun-Shen Hsu, ${ }^{2}$ Tsung-Lun Tsai, ${ }^{2}$ Chia-Ju Liu, ${ }^{2}$ and Ming-Chung Ho ${ }^{1 *}$ \\ ${ }^{1}$ Department of Physics, National Kaohsiung Normal University, \\ No. 62, Shenjhong Rd., Yanchao District, Kaohsiung City 824, Taiwan, R.O.C. \\ ${ }^{2}$ Graduate Institute of Science Education \& Environmental Education, National Kaohsiung Normal University, \\ No. 62, Shenjhong Rd., Yanchao District, Kaohsiung City 824, Taiwan, R.O.C.
}

(Received November 22, 2018; accepted June 20, 2019)

Keywords: Chua's circuit, mutual inductance effect, phase synchronization

In this study, we proposed a novel method that is different from previous methods using feedback to achieve synchronization. Our study was carried out to induce the mutual effect coil inductances of two Chua's circuit systems and achieve phase synchronization by the bidirectional coupling of two Chua's circuit systems. Additionally, analyzing the correlation coefficient vs the inductance spacing, we observed the hysteresis phenomenon and the sensitivity of the systems to various chaotic states that could be applied to some sensors.

\section{Introduction}

In the last couple of decades, the focus of studies of nonlinear dynamic systems was on understanding the mechanisms of chaos synchronization and the stability of chaotic systems. ${ }^{(1-3)}$ In recent years, phase synchronization has become increasingly important, because it is helpful for understanding not only the synchronization of neuronal activities of remote areas in the human brain but also multichannel nonlinear digital communications. ${ }^{(4,5)}$

Furthermore, being one of the easiest entities that can be observed in a chaotic behavior, an electronic circuit is very important in a chaotic system and is widely used. Since Pecora and Carroll published their work on the chaos synchronization phenomenon using the electronic circuit method for the first time, ${ }^{(6,7)}$ people proposed many methods of chaos control, such as driving response, linear variable feedback, adaptive control, and phase synchronization. Among these methods that had their own characteristics and domains of applications, the linear variable feedback mechanism was most often used to achieve the chaos synchronization of circuits. However, it is difficult to make two circuits achieve the synchronization of two chaotic systems. To overcome this difficulty, the coupling method was used.

Here, we study a method that is very different from the general coupling method inputting the driven signal to the response circuit system. We utilized coil inductances connected in Chua's circuits with their mutual effect to achieve the phase synchronization of two systems. The case of double-scroll attractor has been discussed in our conference paper, ${ }^{(8)}$ where we *Corresponding author: e-mail: t1603@nknucc.nknu.edu.tw https://doi.org/10.18494/SAM.2019.2202 
presented various behaviors of phase synchronization induced by varying the inductance spacing. In this study, we selected more appropriate experimental parameters, focused on the mutual inductance effect of different initial states, and discussed the hysteresis phenomena in both cases. Additionally, fine-tuning the resistance of Chua's circuits and varying the inductance spacing were sensitive to various chaotic states that could be applicable to the development of sensors.

\section{Experimental Setup}

Two Chua's circuits, where $2 \mathrm{mH}$ coil inductances, $\mathrm{L}_{1}$ and $\mathrm{L}_{2}$, were connected in two systems individually, were utilized, and the experimental setup is shown in Fig. 1(a). Experimental Chua's systems, which consisted of systems 1 and 2 , containing the variable resistors $R_{1}$ and $\mathrm{R}_{2}$, and two coil inductances erected on a stepping motor driver, were automatically controlled by a computer so that they could precisely approach each other and could be separated. In the experiment, a synchronous sampling data acquisition card was used to capture the $\mathrm{x}$ - and y-signals of two Chua's systems simultaneously, where the sampling rate was $1 \mathrm{MS} / \mathrm{s}$ and the number of samplings was 10000 . On the mutual inductance effect, ${ }^{(9)}$ by varying the inductance spacing $D$ as shown in Fig. 1(b), the phenomena of circuit coupling states, that is, the single- and double-scroll attractors, were observed.

\section{Experimental Results}

Through phase, spectrum analysis, and time series diagrams, various states of coupling processes of the two Chua's chaotic systems were observed. Herein, our study is different from former ones, in that it uses two chaotic systems with particular parameters that do not match with each other to achieve directional or bidirectional coupling. ${ }^{(10)}$ In this study, we adjusted the variable resistors $R_{1}$ and $R_{2}$ of two Chua's circuits to change the states of two chaotic oscillations, made two systems achieve bidirectional coupling, and analyzed the various processes of phase synchronization resulting from the mutual inductance effect at different distances between two coil inductances.

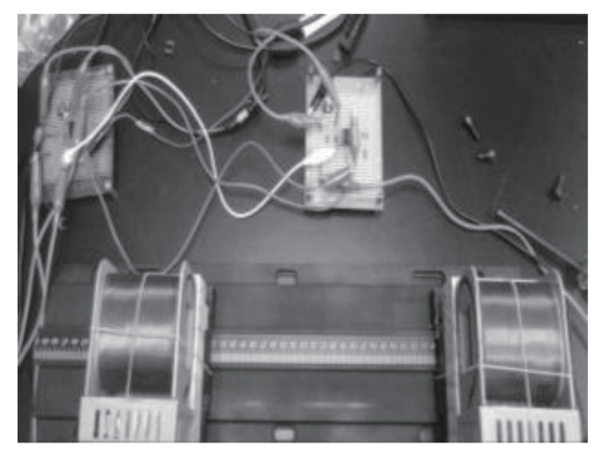

(a)

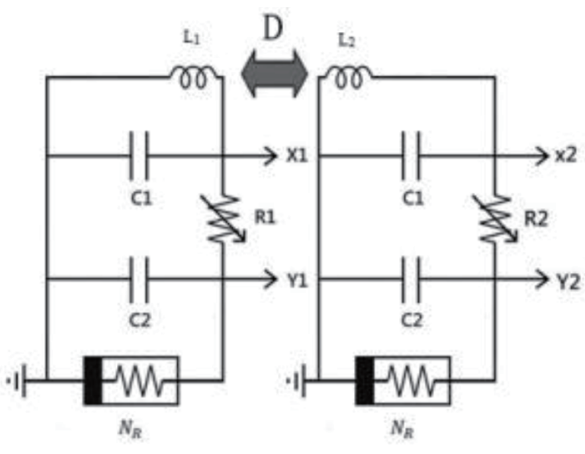

(b)

Fig. 1. (a) Setup and (b) schematic diagram of bidirectional coupling chaotic systems resulting from coil inductances. 


\subsection{Initial states of double-scroll attractors}

First, we adjusted the values of the variable resistors to make the systems appear to be double-scroll attractors as the initial states. Figures 2(a) and 2(b) show the phase diagrams of the initial states of systems 1 and 2, respectively. The results indicate that, when the inductance spacing was $12 \mathrm{~cm}$, there was no mutual response between the two inductances, so no coupling occurred between the two systems. Figures 2(c) and 2(d) show the power spectrum diagrams of the two systems and that the main frequencies of systems 1 and 2 were not identical, indicating that no synchronization occurred. Figure 3 presents the phase diagram of $y_{1} v_{s} y_{2}$, which were

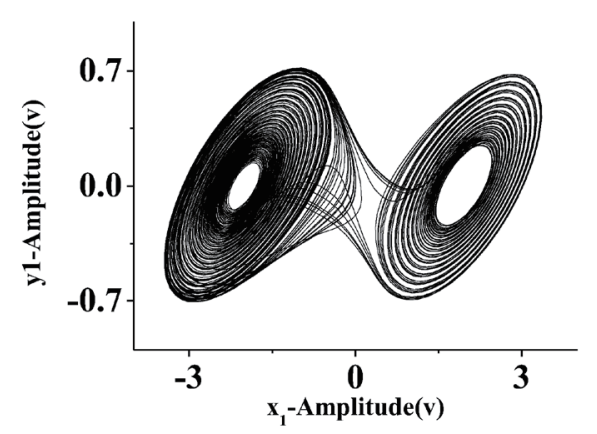

(a)

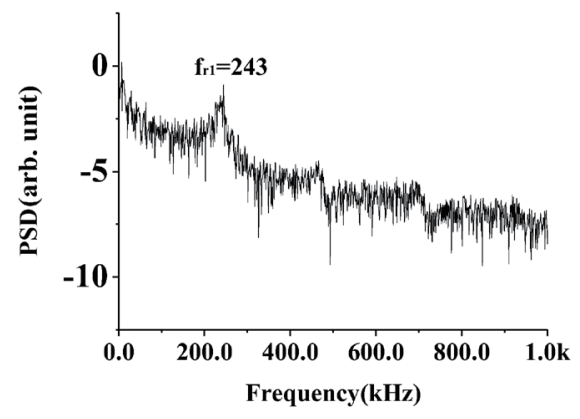

(c)

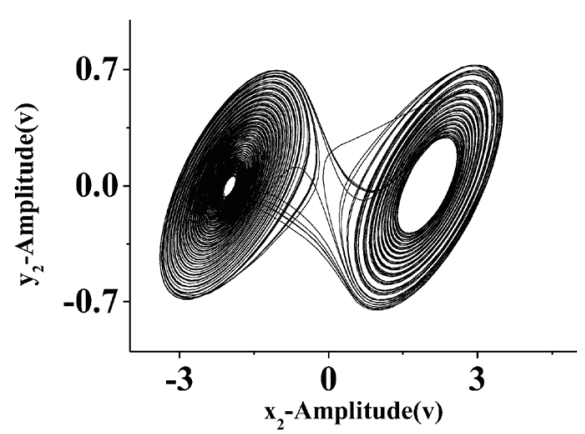

(b)

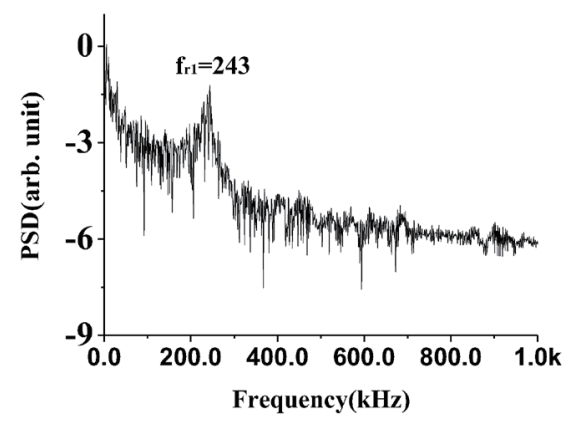

(d)

Fig. 2. Phase diagrams of systems (a) 1 and (b) 2, and power spectrum diagrams of systems (c) 1 and (d) 2 when the inductance spacing was $12 \mathrm{~cm}$, where PSD represented power spectral density.

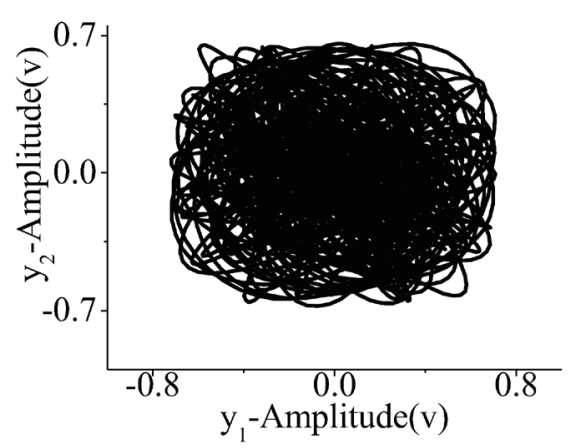

Fig. 3. Phase diagram of $\mathrm{y}_{1}$ vs $\mathrm{y}_{2}$ when the inductance spacing was $12 \mathrm{~cm}$. 
the voltages of systems 1 and 2, respectively. The result shows that the trajectories were chaotic and verifies that the two systems indeed did not achieve synchronization when they were uncoupled.

The mutual inductance effect gradually increased as the two coil inductances approached each other slowly. When the inductance spacing was about $5 \mathrm{~cm}$, the oscillation of systems 1 and 2 turned into a quasi-period state, as shown in Figs. 4(a) and 4(b), from the double-scroll attractor. We analyzed the power spectra of the two systems shown in Figs. 4(c) and 4(d). The main frequencies of systems 1 and 2 were the same at $237 \mathrm{~Hz}$. Then, we plotted the phase diagram of $y_{1} v_{s} y_{2}$, which displayed an inclined line as shown in Fig. 5. The result shows that

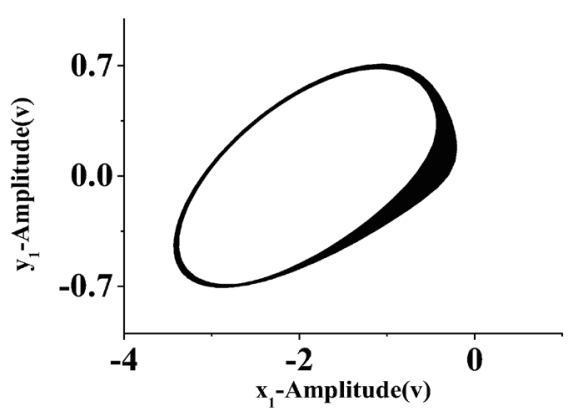

(a)

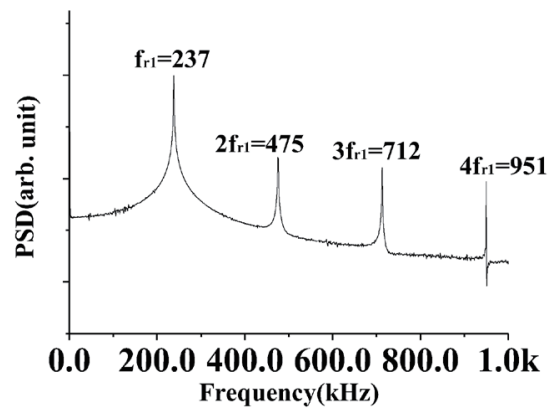

(c)

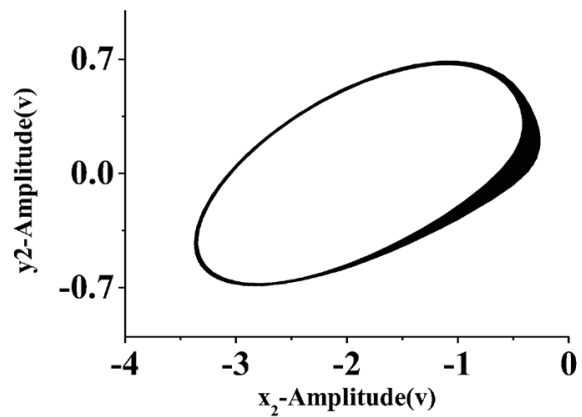

(b)

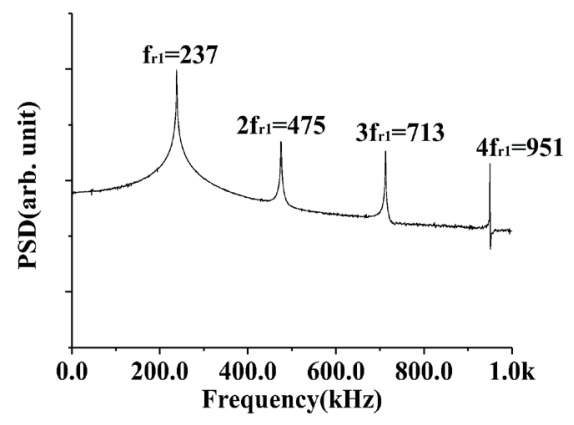

(d)

Fig. 4. Phase diagrams of systems (a) 1 and (b) 2, and power spectra diagrams of systems (c) 1 and (d) 2 when the inductance spacing was $5 \mathrm{~cm}$.

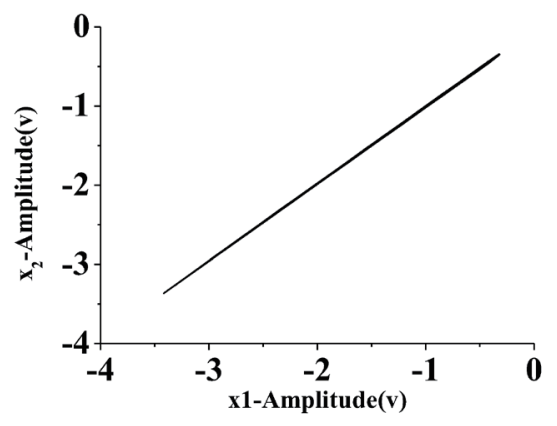

Fig. 5. Phase diagram of $\mathrm{y}_{1}$ vs $\mathrm{y}_{2}$ when the inductance spacing was $5 \mathrm{~cm}$. 
the two systems achieved good phase synchronization because the mutual inductance caused chaos suppression when the inductance spacing was $5 \mathrm{~cm}$.

When the two coil inductances were closer, the states of the two systems changed again. As the inductance spacing continued to decrease from 5 to $1 \mathrm{~cm}$, systems 1 and 2 transformed into double-scroll attractors, as shown in Figs. 6(a) and 6(b), from the quasi-period states, as shown in Figs. 4(a) and 4(b). Power spectrum analysis showed that the main frequencies of the two systems were identical, as shown in Figs. 6(c) and 6(d). The phase diagram of $\mathrm{y}_{1}$ vs $\mathrm{y}_{2}$ displayed the trend of a declined line, although the trajectory was somewhat disordered, as shown in Fig. 7.

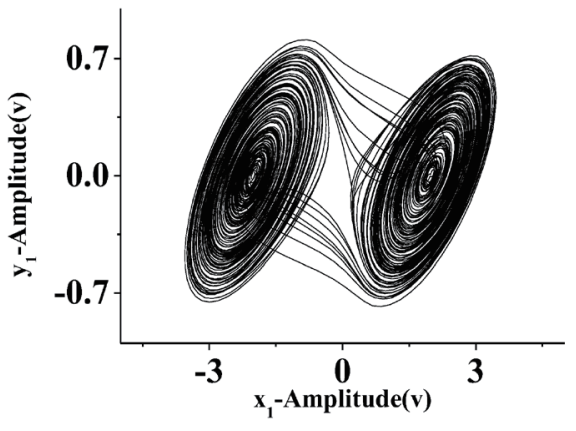

(a)

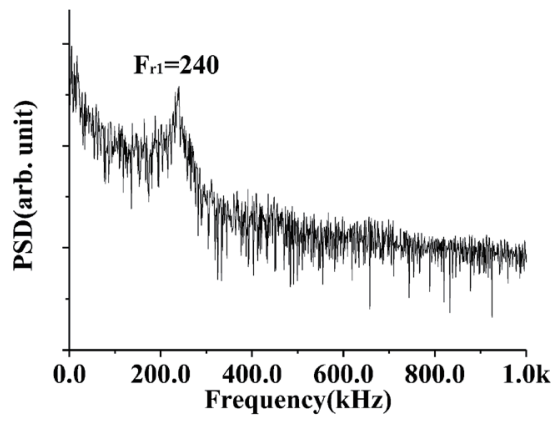

(c)

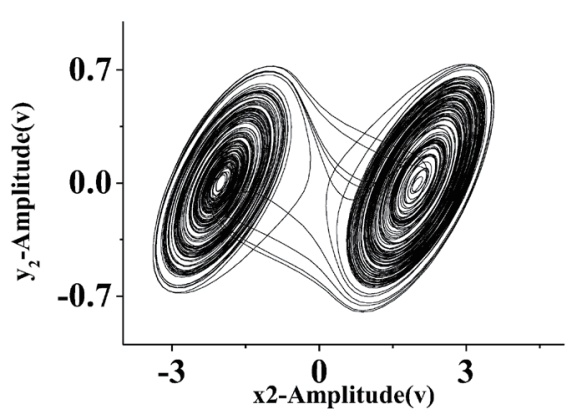

(b)

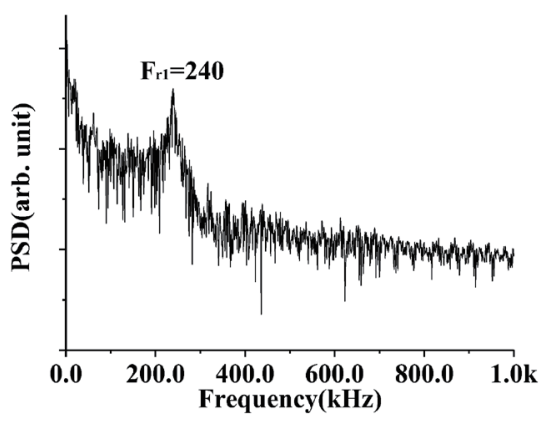

(d)

Fig. 6. Phase diagrams of systems (a) 1 and (b) 2, and power spectrum diagrams of systems (c) 1 and (d) 2 when the inductance spacing was $1 \mathrm{~cm}$.

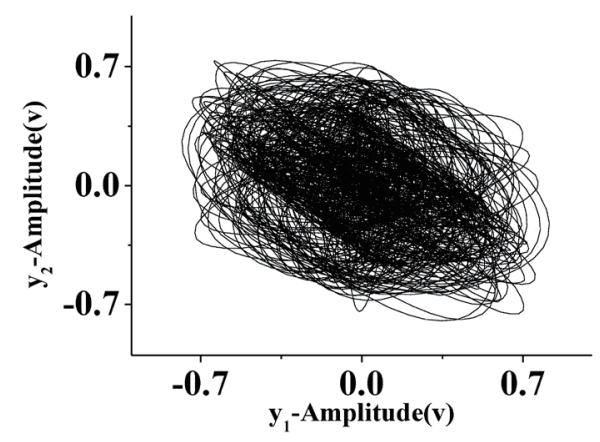

Fig. 7. Phase diagram of $y_{1}$ vs $y_{2}$ when the inductance spacing was $1 \mathrm{~cm}$. 
The result showed that, when the two coil inductances continued to move closer, the two systems would transform into a weak antiphase synchronization from the phase synchronization of chaos suppression. ${ }^{(11)}$

\subsection{Initial states of single-scroll attractors}

When the variable resistor was precisely adjusted to particular values, the chaotic behavior of a single attractor was observed. Thus, we had single-scroll attractors as the initial states of systems 1 and 2, as shown in Figs. 8(a) and 8(b), respectively. Figures 8(c) and 8(d) show the power spectrum diagrams of the two systems, and that the main frequencies of systems 1 and 2 were not identical, that is, no synchronization occurred. Figure 9 shows the phase diagram of

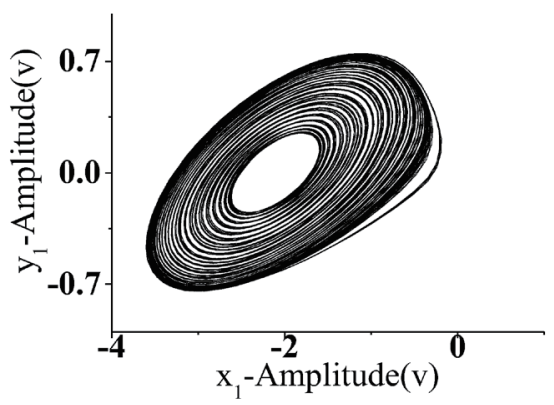

(a)

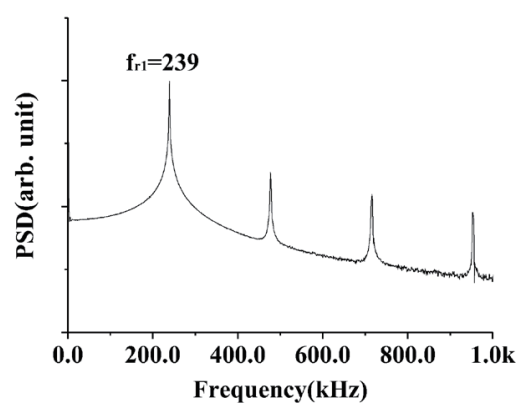

(c)

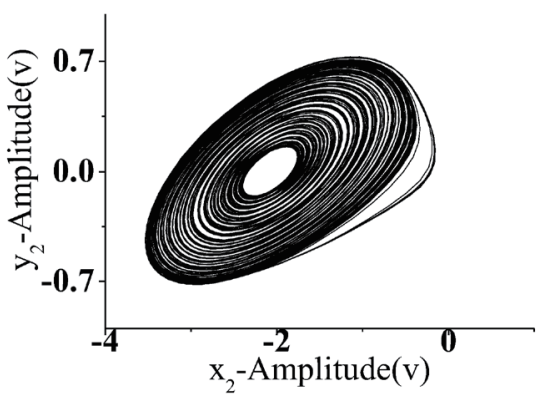

(b)

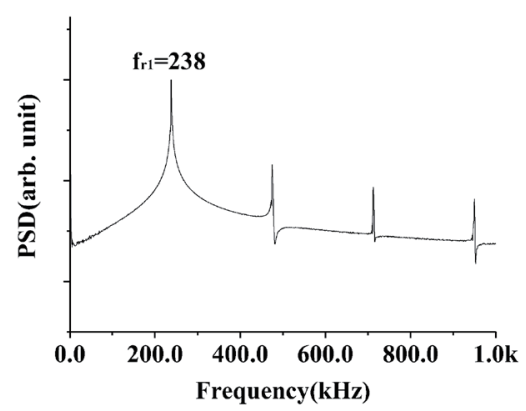

(d)

Fig. 8. Phase diagrams of systems (a) 1 and (b) 2, and power spectrum diagrams of systems (c) 1 and (d) 2 when the inductance spacing was $12 \mathrm{~cm}$.

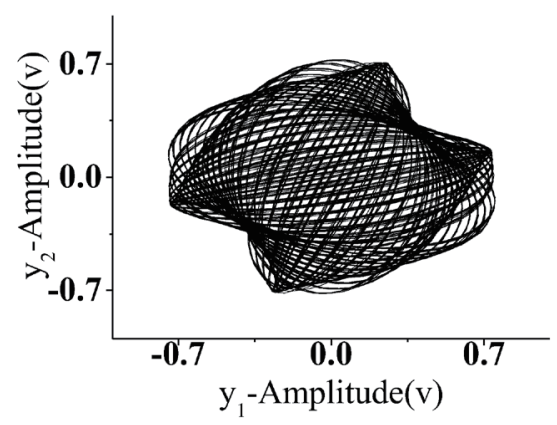

Fig. 9. Phase diagram of $y_{1}$ vs $y_{2}$ when the inductance spacing was $12 \mathrm{~cm}$. 
$\mathrm{y}_{1} \mathrm{vs}_{\mathrm{y}} \mathrm{y}_{2}$. The result shows that the trajectory was disordered, which confirmed that the two systems did not achieve synchronization when they were not coupled.

Then, we reduced the inductance spacing slowly to $6 \mathrm{~cm}$. Although the oscillation states of systems 1 and 2 were the quasi-period state as shown in Figs. 10(a) and 10(b), the power spectrum diagrams revealed that the two main frequencies of systems 1 and 2 were the same, as shown in Figs. 10(c) and 10(d), respectively. Additionally, the phase diagram of $y_{1}$ vs $y_{2}$ shown in Fig. 11 indicates that systems 1 and 2 were synchronized.

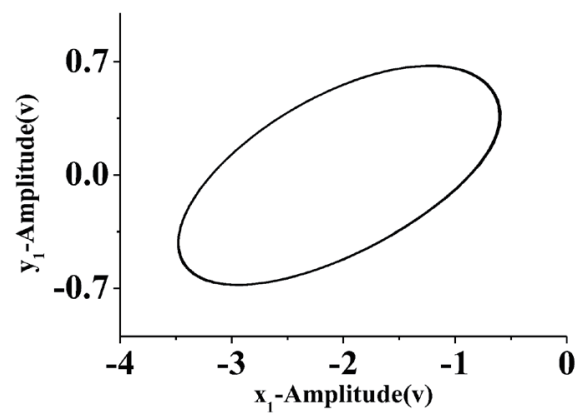

(a)

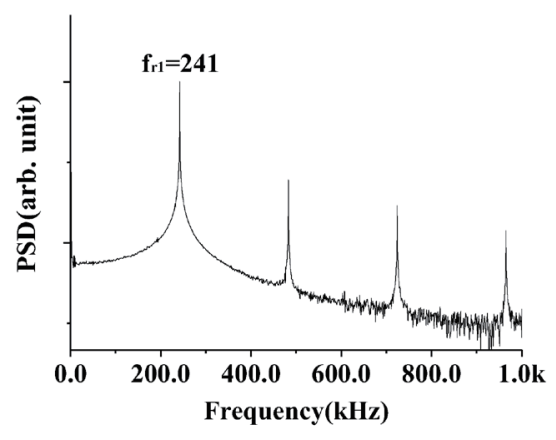

(c)

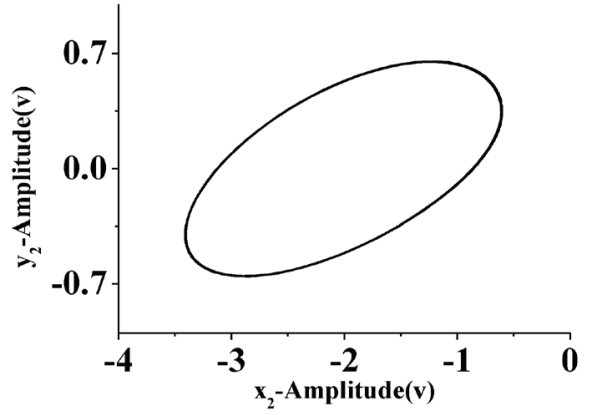

(b)

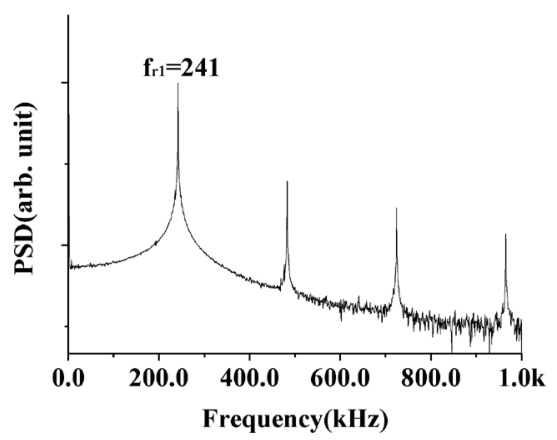

(d)

Fig. 10. Phase diagrams of systems (a) 1 and (b) 2, and power spectrum diagrams of systems (c) 1 and (d) 2 when the inductance spacing was $6 \mathrm{~cm}$.

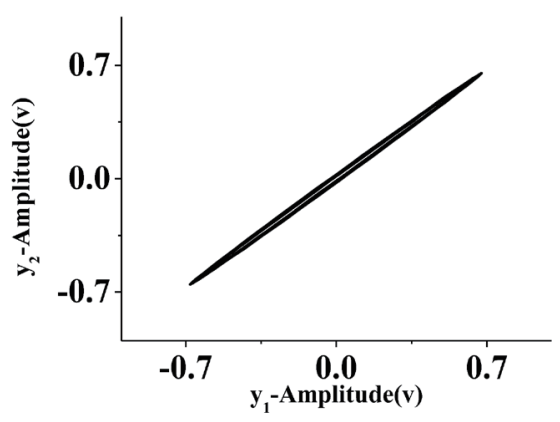

Fig. 11. Phase diagram of $y_{1} v_{s} y_{2}$ when the inductance spacing was $6 \mathrm{~cm}$. 
Similarly, when the inductance spacing continued to decrease from 6 to $2 \mathrm{~cm}$, both systems 1 and 2 transformed into the double-scroll attractors shown in Figs. 12(a) and 12(b) from the quasi-period states shown in Figs. 10(a) and 10(b). Power spectrum analysis showed that the main frequencies of the two systems were identical, as shown in Figs. 12(c) and 12(d). The phase diagram of $y_{1}$ vs $y_{2}$ displayed the trend of a declined line, although the trajectory was somewhat disordered, as shown in Fig. 13. The result showed that the two systems achieved a weak antiphase synchronization when the two coil inductances continued to move closer.

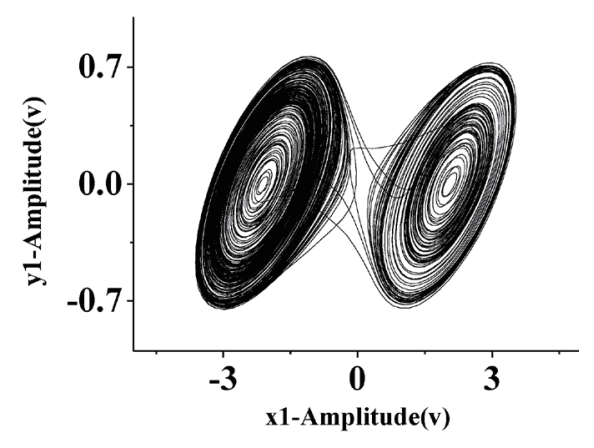

(a)

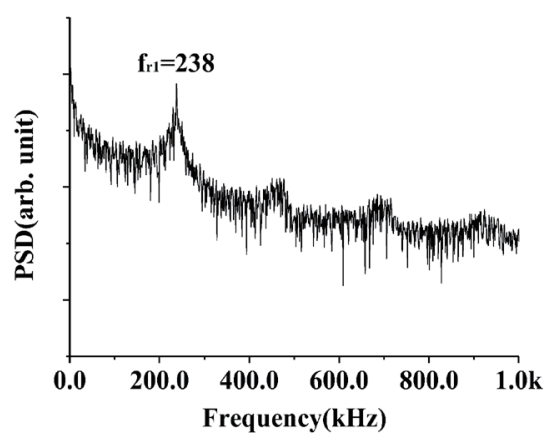

(c)

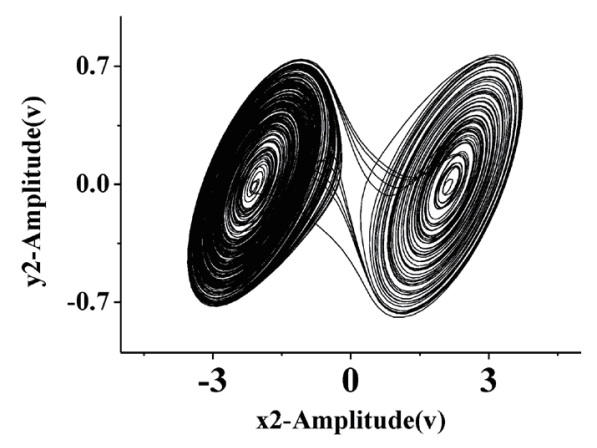

(b)

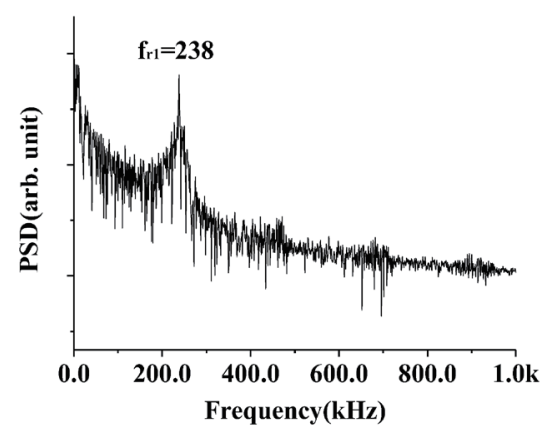

(d)

Fig. 12. Phase diagrams of systems (a) 1 and (b) 2, and power spectrum diagrams of systems (c) 1 and (d) 2 when the inductance spacing was $2 \mathrm{~cm}$.

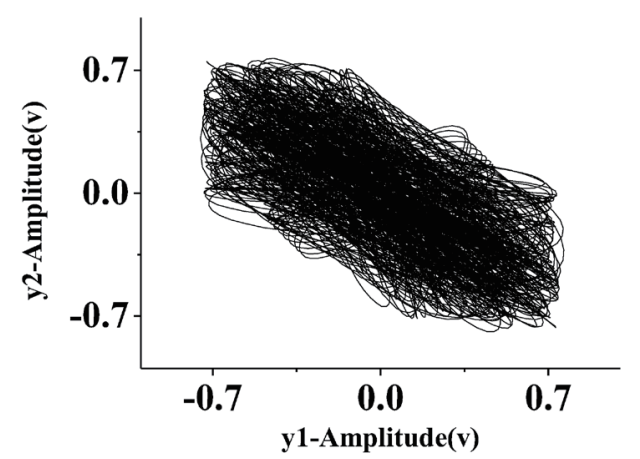

Fig. 13. Phase diagram of $y_{1} v s y_{2}$ when the inductance spacing was $2 \mathrm{~cm}$. 


\section{Discussion}

Experimental results revealed that, when two coil inductances moved close to an appropriate distance, the two systems would transform from being originally incoherent to showing a better phase synchronization. Additionally, when the two coil inductances continued to move closer, the two systems would transform from showing a better phase synchronization to showing a weak antiphase synchronization instead. In view of this, we were interested in synchronous changes between the two systems at different inductance spacings. Therefore, we further carried out the correlation analysis of the two systems at various inductance spacings.

First, in the case of double-scroll attractors as the initial states, we measured the correlation coefficients without delay time when the inductance spacing was changed from 8 to $0 \mathrm{~cm}$, as shown in Fig. 14, where black lines represent the process in which the two inductances approach closer to each other (approaching process) and gray lines the separation process. From 8 to about $1.1 \mathrm{~cm}$, most of the correlation coefficients fluctuated around zero, indicating the absence of synchronization. From 1.1 to $0.6 \mathrm{~cm}$, most of the correlation coefficients reached 1, indicating good synchronization. From 0.6 to $0 \mathrm{~cm}$, most of the correlation coefficients decreased to -0.5 , indicating a weak antisynchronization. Then, in the case of single-scroll attractors as the initial states, we measured the correlation coefficients without delay time when the inductance spacing was changed from 8 to $0 \mathrm{~cm}$, as shown in Fig. 15, where black lines represent the approaching process and gray lines the separation process. From 8 to about $5 \mathrm{~cm}$, most of the correlation coefficients fluctuated around zero, indicating the absence of synchronization. From 5 to $1.9 \mathrm{~cm}$, most of the correlation coefficients reached 1, indicating good synchronization. From 1.3 to $0 \mathrm{~cm}$, most of the correlation coefficients decreased to -0.5 , indicating a weak antisynchronization.

In addition, we also measured the correlation coefficients of the two coil inductances in the approaching and separation processes. Figures 14 and 15 show new findings, that is, the trajectories of the approaching and separating processes did not match, which was caused by the magnetic hysteresis resulting from the experimental inductance of the coils. ${ }^{(12)}$ Moreover,

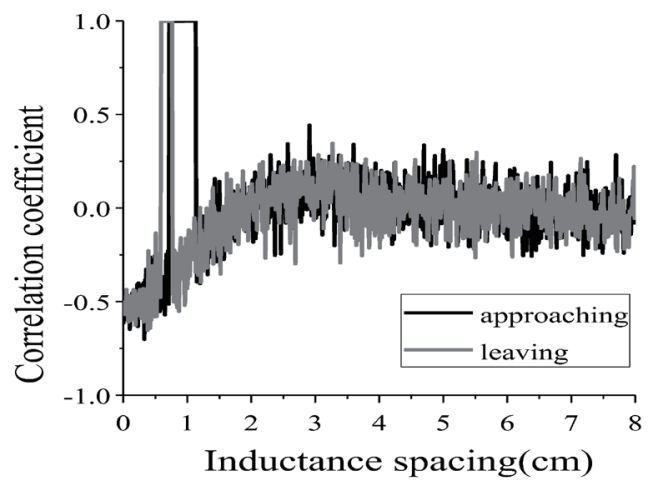

Fig. 14. In the case of double-scroll attractors as the initial states, the diagram of correlation coefficients vs inductance spacing is shown.

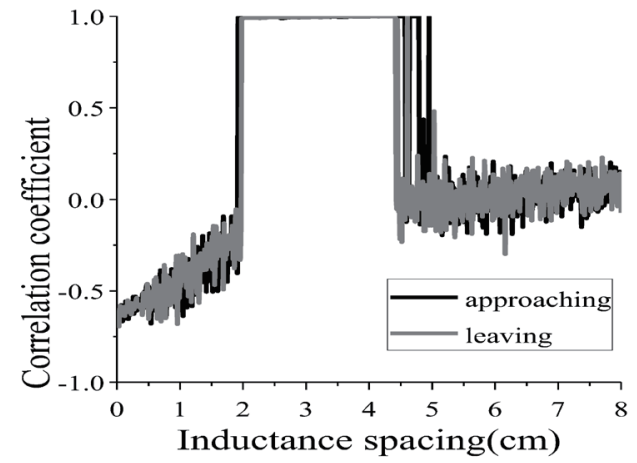

Fig. 15. In the case of single-scroll attractors as the initial states, the diagram of correlation coefficients vs inductance spacing is shown. 
in Figs. 14 and 15, we also observed that there was a wider interval of chaos suppression in the case of single-scroll attractors as the initial states than in that of double-scroll attractors as the initial states, and that there was also a wider interval of chaos suppression in the approaching process than in the separation process.

\section{Conclusions}

The mutual inductance effect increased with the decrease in spacing between the two coil inductances, and the coupling strength of two Chua's systems increased with the increase in the mutual inductance effect. Good chaotic suppression occurred when the coupling strength increased to certain values. However, when the coupling strength continued to increase, the synchronization decreased instead, and a weak antisynchronization was observed.

In this study, the synchronization resulting from external coil inductances, which has never been proposed to date, was quite different from the previous ones that resulted from the inner controller's input to the systems. It is possible to develop an external mechanic key for encrypting and decrypting, which was harder to crack.

\section{References}

1 A. S. Pikovsky, M. Rosenblum, and J. Kurths: Synchronization: A Universal Concept in Nonlinear Science (Cambridge University Press, New York, 2001) p. 141.

2 S. Boccaletti, V. Latora, Y. Moreno, M. Chavez, and D.-U. Hwang: Phys. Rep. 424 (2006) 175. http://doi. org/10.1016/j.physrep.2005.10.009

3 L. M. Pecora and T. L. Carroll: Interdiscip. J. Nonlinear Sci. 25 (2015) 097611. http://doi.org/10.1063/1.4917383

4 P. Tass, M. G. Rosenblum, J. Weule, J. Kurths, A. Pikovsky, J. Volkmann, A. Schnitzler, and H. J. Freund: Phys. Rev. Lett. 81 (1998) 3291. http://doi.org/10.1103/physrevlett.81.3291

5 T. Yalçlnkaya and Y. C. Lai: Phys. Rev. A 79 (1997) 3885. http://doi.org/10.1103/physrevlett.79.3885

6 L. M. Pecora and T. L. Carroll: Phys. Rev. Lett. 64 (1990) 821. https://doi.org/10.1103/PhysRevLett.64.821

7 T. L. Carroll and L. M. Pecora: IEEE Trans. Circuits Syst. 38 (1991) 453. https://doi.org/10.1109/31.75404

8 C. J. Liu, F. W. Tsai, C. S. Hsu, J. C. Lee, M. C. Lu, C. A. Chan, and M. C. Ho: IEEE-ICAMSE (2016) 235. http://doi.org/10.1109/icamse.2016.7840296

9 H. Pahlavani and E. Rahmanpour Kolur: Physica B 495 (2016) 123. https://doi.org/10.1016/j.physb.2016.05.009

10 B. Cannas and S. Cincotti: Int. J. Circuit Theory Appl. 30 (2002) 625. https://doi.org/10.1002/cta.213

11 C. M. Kim, S. Rim, W. H. Kye, J. W. Ryu, and Y. J. Park: Phys. Lett. A 320 (2003) 39. http://doi.org/10.1016/ j.physleta.2003.10.051

12 D. C. Jiles and D. L. Atherton: IEEE Trans. Magn. 19 (1983) 2183. http://doi.org/10.1109/TMAG.1983.1062594 